\title{
Effects of Electron Correlation and Scalar Relativistic Corrections on the Thermochemical and Spectroscopic Properties of HOF
}

\author{
B. Ramachandran, ${ }^{* \dagger}$ Naga Srivani Vegesna, ${ }^{\dagger}$ and Kirk A. Peterson ${ }^{\S}$ \\ Chemistry, College of Engineering and Science, Louisiana Tech University, Ruston, Louisiana 71272, and \\ Department of Chemistry, Washington State University, Pullman, Washington 99164-4630
}

Received: May 8, 2003; In Final Form: July 28, 2003

\begin{abstract}
Bond dissociation energies for the hypofluorous acid (HOF) system and an anharmonic force field for HOF are obtained from ab initio energies at the CCSD(T)/CBS level of theory, where the complete basis set (CBS) limit energies are obtained by extrapolating $\operatorname{CCSD}(\mathrm{T}) /$ aug-cc-pVnZ $(n=2,3$, and 4$)$ energies. The effects of including core-valence correlation and scalar relativistic effects on the thermochemical and spectroscopic properties are investigated. The results are compared to available experimental results including recent reevaluations of the properties of $\mathrm{OF}$ and $\mathrm{OH}$. Quartic force fields are calculated at each level of theory and basis set used. Excellent agreement between experimental and calculated properties is obtained in most cases, but a few prominent and large differences in rotation-vibration coupling constants and a few force constants are observed. In these cases, our results are consistent with other, high-level, ab initio evaluations of these properties. Given the ability of large basis set $\operatorname{CCSD}(\mathrm{T})$ calculations to yield accurate results for a wide range of molecular parameters, these differences suggest that experimental results may benefit from reexamination in some cases. A new recommendation for the $0 \mathrm{~K}$ enthalpy of formation of $\mathrm{HOF}, \Delta H_{f, 0 \mathrm{~K}}^{\circ}=$ $-20.02 \pm 0.25 \mathrm{kcal} / \mathrm{mol}$, is presented based on a careful analysis of recent experimental data and is supported by the high-level ab initio calculations presented in this work.
\end{abstract}

\section{Introduction}

There have been many determinations of the equilibrium structure of hypofluorous acid (HOF) using $\mathrm{SCF}^{1-4}$ and various correlated methods, ${ }^{5-13}$ including density functional theory. ${ }^{9,10}$ However, there are remarkably few theoretical investigations of the spectroscopic constants, anharmonic force field, and nearequilibrium potential energy function of the HOF molecule, despite the availability of a rather large array of experimental results. ${ }^{14-22}$ The earliest attempts in this direction appear to be those of Botschwina ${ }^{2}$ and Murrell et al. ${ }^{4}$ The former ${ }^{2}$ reported calculations of a near-equilibrium potential energy surface at the SCF level of theory coupled with empirical corrections to the quadratic force field to improve the predictions of higherlying overtones and combination bands. The latter ${ }^{4}$ described a global potential energy surface derived partially from a quadratic force field calculated from experimental vibrational frequencies and partially from SCF calculations. The first calculation of spectroscopic properties and an anharmonic force field for $\mathrm{HOF}$ that included electron correlation effects appears to be the coupled cluster singles and doubles (CCSD) investigations of Thiel et al. ${ }^{23}$ Halonen and $\mathrm{Ha}^{20}$ constructed an empirical quartic force field by a constrained least-squares fit to available experimental vibration-rotation data supplemented by several $\mathrm{ab}$ initio force constants from Thiel et al. The most accurate theoretical calculations of the spectroscopic constants and anharmonic force field of HOF to date appears to be that of Breidung et al., ${ }^{24}$ who used the CCSD method with perturbative triples, $\operatorname{CCSD}(\mathrm{T}),{ }^{25,26}$ making use of the then-recently implemented analytic second derivatives ${ }^{27}$ to obtain a quartic force

* Corresponding author. E-mail: ramu@latech.edu.

† Louisiana Tech University.

$\S$ Washington State University. field. To the best of our knowledge, refs 23 and 24 represent the only reliable theoretical estimates of the spectroscopic constants of the HOF molecule available at this time.

The present work was undertaken with two goals. One was to examine the accuracy with which "high level" (to be defined below) ab initio calculations can predict the thermochemistry of the $X^{1} A^{\prime}$ electronic state of the "HOF system," by which we mean the triatomic molecule and the atom-diatom fragments that correlate with it. This was motivated by a recent reevaluation of the spectroscopic constants of the OF radical from available high-resolution experimental data, ${ }^{28}$ the dissociation energy of $\mathrm{OH}$ from a combination of experimental data and large-scale ab initio calculations, ${ }^{29}$ and a suspected uncertainty of almost $3.0 \mathrm{kcal} / \mathrm{mol}$ in the enthalpy of formation at $0 \mathrm{~K}$, $\Delta H_{f, 0 \mathrm{~K}}^{\circ}$, of HOF. ${ }^{30}$ The second goal was to examine the spectroscopic constants of $\mathrm{HOF}, \mathrm{OH}, \mathrm{OF}$, and $\mathrm{HF}$ molecules from local analytic potential energy surfaces constructed from the above-mentioned "high level" ab initio energies.

By "high level," we mean ab initio calculations at the CCSD(T) level of theory coupled with (a) the recovery of correlation energy beyond that afforded by standard correlation consistent basis sets by extrapolation to the complete basis set (CBS) limit, (b) inclusion of core-valence (CV) correlation effects, and (c) inclusion of scalar relativistic (SR) effects.

The remainder of this paper is organized as follows. In the following section, we present the details of the calculations. The results of these calculations are presented in Section III and are compared to available experimental results. Section IV is a summary of this work.

\section{Calculations}

The vast majority of calculations were done using the CCSD(T) method with the augumented, correlation consistent, polar- 
TABLE 1. Born-Oppenheimer Dissociation Energies $D_{\mathrm{e}}$ of the HOF System ${ }^{a}$

\begin{tabular}{lrrrrrrr}
\hline & AVDZ & AVTZ & AVQZ & CBS & CBS + CV & CBS + CV + SR & expt $^{b}$ \\
\hline $\mathrm{HF}\left({ }^{1} \sum^{+}\right) \rightarrow \mathrm{H}\left({ }^{2} S\right)+\mathrm{F}\left({ }^{2} P\right)$ & 134.45 & 139.29 & 140.93 & 141.88 & 142.05 & 141.85 & $141.46 \pm 0.2^{c}$ \\
$\mathrm{OH}\left({ }^{2} \Pi\right) \rightarrow \mathrm{H}\left({ }^{2} S\right)+\mathrm{O}\left({ }^{3} P\right)$ & 99.86 & 104.28 & 106.28 & 107.15 & 107.20 & 107.08 & $107.15 \pm 0.07^{d}$ \\
$\mathrm{OF}\left({ }^{2} \Pi\right) \rightarrow \mathrm{O}\left({ }^{3} P\right)+\mathrm{F}\left({ }^{2} P\right)$ & 42.05 & 49.22 & 51.08 & 52.08 & 52.07 & 52.02 & $53.37 \pm 2.26^{e}$ \\
$\mathrm{HOF} \rightarrow \mathrm{O}\left({ }^{3} P\right)+\mathrm{HF}$ & 9.03 & 14.38 & 15.60 & 16.23 & 16.21 & 16.23 & $16.41 \pm 0.25^{f}$ \\
$\mathrm{HOF} \rightarrow \mathrm{O}\left({ }^{1} D\right)+\mathrm{HF}^{g}$ & 54.40 & 59.75 & 60.97 & 61.60 & 61.58 & 61.60 & $61.78 \pm 0.25^{f}$ \\
$\mathrm{HOF} \rightarrow \mathrm{F}+\mathrm{OH}$ & 43.62 & 48.94 & 50.25 & 50.95 & 51.06 & 51.00 & $50.94 \pm 0.25^{f}$ \\
$\mathrm{HOF} \rightarrow \mathrm{H}+\mathrm{OF}$ & 101.43 & 104.45 & 105.46 & 106.04 & 106.31 & 106.19 & $104.77 \pm 0.25^{f}$
\end{tabular}

${ }^{a}$ All energies are in $\mathrm{kcal} / \mathrm{mol}$. Spin-orbit splittings have been removed from experimental energies shown. ${ }^{b}$ The experimental values are corrected for spin-orbit splittings since the ab initio calculations do not include them. The corrections used are: $\mathrm{O}\left({ }^{3} P\right)$ : $0.216 \mathrm{kcal} / \mathrm{mol}(\mathrm{ref} 41), \mathrm{F}\left({ }^{2} P\right)$ : $0.385 \mathrm{kcal} / \mathrm{mol}(\operatorname{ref} 41), \mathrm{OH}\left({ }^{2} \Pi\right): 0.109 \mathrm{kcal} / \mathrm{mol}(\operatorname{ref} 29)$, and $\mathrm{OF}\left({ }^{2} \Pi\right): 0.281 \mathrm{kcal} / \mathrm{mol}(\mathrm{ref} 28) .{ }^{c}$ Calculated from the $D_{0} \mathrm{value}$ given in ref 42 . ${ }^{d}$ Reference 29. ${ }^{e}$ Reference 52. ${ }^{f}$ Based on the "best experimental value" for the $\Delta H_{f, 0 \mathrm{~K}}^{\circ}$ of HOF, $-20.02 \pm 0.25 \mathrm{kcal} / \mathrm{mol}$ (see text). ${ }^{g} \mathrm{The}$ singlet triplet splitting is fixed at the experimental value of $45.366 \mathrm{kcal} / \mathrm{mol}$ in all cases.

ized valence $n$-zeta basis sets of Dunning and co-workers, ${ }^{31}$ (denoted as aug-cc-pVnZ or $\mathrm{AV} n \mathrm{Z}$ ), where $n=2,3$, and 4 (D, T, and Q). Thus, three successively larger calculations were carried out at each geometry of interest. The three $\mathrm{AVnZ}$ energies corresponding to each geometry were then extrapolated to the CBS limit using the expression ${ }^{32}$

$$
E(n)=E_{\mathrm{CBS}}+B \mathrm{e}^{-(n-1)}+C \mathrm{e}^{-(n-1)^{2}}
$$

With the three $\operatorname{CCSD}(\mathrm{T}) / \mathrm{AV} n \mathrm{Z}$ energies corresponding to $n=$ 2, 3, and 4, this leads to three simultaneous equations which are solved for the three unknowns $E_{\mathrm{CBS}}, B$, and $C$.

The core-valence correlation energy is obtained from the difference of frozen-core (fc) and all-electron (ae) energies calculated using the cc-pCVTZ (CVTZ) basis set ${ }^{33}$

$$
\delta E_{\mathrm{CV}}=\left(E_{\mathrm{CVTZ}}^{\mathrm{CCSD}(\mathrm{T}) / \mathrm{ae}}-E_{\mathrm{CVTZ}}^{\mathrm{CCSD}(\mathrm{T}) / \mathrm{fc}}\right)
$$

Note that since the all-electron energy is lower than that of the frozen-core calculation, the left-hand side of eq 2 is negative. The scalar relativistic corrections $\delta E_{\mathrm{SR}}$ are obtained as the expectation value of the 1-electron mass velocity and Darwin operators with internally contracted, averaged coupled pair functional (ACPF) wave functions, ${ }^{34}$ where full valence complete active space reference functions were used.

To facilitate discussion of the results in the following section, we define energies at the following levels of theory in addition to the $E_{\mathrm{AV} n \mathrm{Z}}$ and $E_{\mathrm{CBS}}$ levels. These are

$$
E_{\mathrm{CBS}+\mathrm{CV}}=E_{\mathrm{CBS}}+\delta E_{\mathrm{CV}}
$$

and

$$
E_{\mathrm{CBS}+\mathrm{CV}+\mathrm{SR}}=E_{\mathrm{CBS}}+\delta E_{\mathrm{CV}}+\delta E_{\mathrm{SR}}
$$

All calculations were carried out using the MOLPRO package of programs,${ }^{35}$ and in all cases only the pure spherical harmonic components of the angular momentum functions in the basis set were used. For the various open-shell species involved, the R/UCCSD(T) method was used. ${ }^{36}$

To construct near-equilibrium potential energy surfaces for the HOF molecule, a total of 41 points in $\left(r_{\mathrm{OH}}, r_{\mathrm{OF}}, \theta_{\mathrm{HOF}}\right)$ space spanning the experimental equilibrium geometry were computed at the $\operatorname{CCSD}(\mathrm{T})$ level of theory using each of the AVnZ $(n=$ $\mathrm{D}, \mathrm{T}, \mathrm{Q}$ or 2, 3, 4) basis sets, extrapolated to the CBS limit using eq 1, and the core-valence and scalar relativistic corrections were applied to this energy. These energies were then fitted to a polynomial expansion using displacement coordinates for the two bond lengths and the Carter-Handy coordinate $^{37}$ for the bend, using the program SURFIT. ${ }^{38} \mathrm{~A}$ fourth-order expansion in all coordinates was used along with fifth- and sixth-order "diagonal" terms in each coordinate, to yield a total of 41 linear parameters to be determined by the fitting routines. Since the number of parameters and the number of dependent variables are the same, the fit is, in reality, an interpolation, essentially free of statistical error in every case. The equilibrium geometry reported for the molecule corresponds to the location of the minimum on this potential surface.

The SURFIT program transforms the coefficients of the polynomial expansion of the potential energy function into dimensionless normal coordinate coefficients using the $L$-tensor algebra of Hoy, Mills, and Strey. ${ }^{39}$ The resulting normal mode expansion coefficients are used to compute the spectroscopic constants using standard second-order perturbation theory expressions ${ }^{40}$ for the ro-vibrational energy levels of the molecule. The force field can also be obtained in terms of massindependent internal coordinates in the usual Taylor series expansion about the equilibrium geometry.

The spectroscopic properties of the diatomic molecules were analyzed using a sixth-order polynomial interpolation through seven ab initio points from which all the required derivatives were evaluated analytically. The reported equilibrium geometries for the diatomics correspond to the minima of these potential energy curves.

\section{Results and Discussion}

III.A. Dissociation Energies. The Born-Oppenheimer dissociation energies $D_{\mathrm{e}}$ of the HOF system are presented in Table 1 at the various levels of theory used and compared to experimental values. Since the ab initio calculations do not include spin-orbit coupling, the experimental values used for comparison have been adjusted using experimental atomic ${ }^{41}$ and molecular ${ }^{28,29}$ spin-orbit splittings, and therefore, the comparisons are meaningful.

The experimental $D_{\mathrm{e}}$ values reported for the dissociation of the HOF molecule into atom + diatom combinations deserve comment. The $\Delta H_{f, 0 \mathrm{~K}}^{\circ}$ of HOF from the JANAF Tables ${ }^{42}$ is $-22.8 \pm 1.00 \mathrm{kcal} / \mathrm{mol}$. However, Pople and Curtiss ${ }^{30}$ have expressed the opinion that this value is in error and recommended $-19.9 \mathrm{kcal} / \mathrm{mol}$ as the "best" experimental value, obtained by combining the photoionization process ${ }^{43}$

$$
\mathrm{HOF}+h v \rightarrow \mathrm{OH}^{+}+\mathrm{F}+\mathrm{e}^{-}
$$

for which $\Delta H_{f, 0 \mathrm{~K}}^{\circ}=15.07 \pm 0.01 \mathrm{eV}$ (347.52 \pm 0.23 $\mathrm{kcal} / \mathrm{mol}),{ }^{44}$ with the ionization energy (IE) and the $\Delta H_{f, 0 \mathrm{~K}}^{\circ}$ of $\mathrm{OH}$ and $\mathrm{F}\left({ }^{2} P\right)$. By combining eq 5 with the ionization of $\mathrm{OH}$, we get

$$
\mathrm{HOF} \rightarrow \mathrm{OH}\left({ }^{2} \Pi\right)+\mathrm{F}\left({ }^{2} P\right)
$$

If the $0 \mathrm{~K}$ enthalpies of formation of $\mathrm{OH}$ and $\mathrm{F}\left({ }^{2} P\right)$ are available, 
$\Delta H_{f 0 \mathrm{~K}}^{\circ}$ for HOF can, therefore, be easily calculated. For IE$(\mathrm{OH})$, Pople and Curtiss used the Katsumata-Lloyd value of $13.01 \mathrm{eV}^{45}$, and for the $0 \mathrm{~K}$ enthalpies of formation of $\mathrm{OH}$ and $\mathrm{F}\left({ }^{2} P\right)$, they used the JANAF Table values of $9.175 \pm 0.29 \mathrm{kcal} /$ mol and $18.47 \pm 0.072 \mathrm{kcal} / \mathrm{mol}$, respectively. Two of these values have since been called into question. We refer the reader to ref 29 for a brief history of the measurement of the ionization potential of $\mathrm{OH}$. The Katsumata-Lloyd value is certainly among the more accurate measurements, but it seems that the most accurate adiabatic ionization energy of $\mathrm{OH}$ to date is from the zero-electron kinetic energy (ZEKE) photoionization study of Weidmann et al., ${ }^{46}$ which is reported to be $13.01698 \pm 0.00025$ $\mathrm{eV}$. Using this result, the $0 \mathrm{~K}$ enthalpy of reaction, $\Delta H_{r, 0 \mathrm{~K}}^{\circ}$ for eq 6 is $2.05 \pm 0.01 \mathrm{eV}$ or $47.34 \pm 0.23 \mathrm{kcal} / \mathrm{mol}$. A recommendation for the $\Delta H_{f, 0 \mathrm{~K}}^{\circ}$ for $\mathrm{HOF}$ of $-19.5 \mathrm{kcal} / \mathrm{mol}$ has been made recently, ${ }^{47}$ using this $\Delta H_{r, 0 \mathrm{~K}}^{\circ}$ for eq 6 and the JANAF values for the $\Delta H_{f, 0 \mathrm{~K}}^{\circ}$ of $\mathrm{OH}$ and $\mathrm{F}\left({ }^{2} P\right) .{ }^{48}$ However, the recent report of Ruscic et al. ${ }^{29}$ lends strong support for revising the $0 \mathrm{~K}$ enthalpy of formation of $\mathrm{OH}$ to $8.85 \pm 0.07 \mathrm{kcal} / \mathrm{mol}$. With the $\Delta H_{r .0 \mathrm{~K}}^{\circ}$ of $47.34 \pm 0.23 \mathrm{kcal} / \mathrm{mol}$ for eq 6 , the Ruscic et al. ${ }^{29}$ value for the $\Delta H_{f, 0 \mathrm{~K}}^{\circ}$ of $\mathrm{OH}$, and adopting the JANAF value for the $\Delta H_{f, 0 \mathrm{~K}}^{\circ}$ for $\mathrm{F}\left({ }^{2} P\right)$, we obtain the "best" experimental value for the $\Delta H_{f, 0 \mathrm{~K}}^{\circ}$ of $\mathrm{HOF}$ to be $-20.02 \pm 0.25 \mathrm{kcal} /$ mol. This is the basis for the experimental $D_{\mathrm{e}}$ values reported for the dissociation of the HOF molecule reported in Table 1.

Table 1 shows that, as expected, the $D_{\mathrm{e}}$ values increase with increasing basis set size and on extrapolation to the CBS limit. The addition of core-valence correlation and scalar relativistic corrections, however, are observed to either increase or decrease the $D_{\mathrm{e}}$ values compared to the valence-only CBS values. So, for instance, the inclusion of core-valence correlation in HF, $\mathrm{F}\left({ }^{2} P\right)$, and $\mathrm{O}\left({ }^{3} P\right)$ results in a net increase in the dissociation energy of $\mathrm{HF}$, but the same treatment slightly decreases the $D_{\mathrm{e}}$ value for the $\mathrm{OF}$ molecule. In nearly every case, the inclusion of scalar relativistic corrections results in a decrease of the $D_{\mathrm{e}}$ value compared to the $\mathrm{CBS}+\mathrm{CV}$ level of theory, the only exception being the $\mathrm{HOF} \rightarrow \mathrm{HF}+\mathrm{O}\left({ }^{3} P\right)$ dissociation, in which case the $\mathrm{CBS}+\mathrm{CV}+\mathrm{SR}$ level essentially recovers the $D_{\mathrm{e}}$ calculated at the CBS level. With two notable exceptions, which will be discussed further below, the AVQZ and higher-level results are in excellent agreement with the experimental values, the differences being much smaller than the $\pm 1 \mathrm{kcal} / \mathrm{mol}$ error limit generally considered sufficient for "chemical accuracy".

It should be mentioned here that since $\operatorname{CCSD}(\mathrm{T})$ does not accurately reproduce the triplet-singlet gap in atomic oxygen (this requires at least a two-determinant treatment), we have fixed this gap to the experimental value of $45.366 \mathrm{kcal} / \mathrm{mol}$ (corrected for SO splitting) when calculating the $D_{\mathrm{e}}$ values for $\mathrm{HOF} \rightarrow \mathrm{O}\left({ }^{1} D\right)+\mathrm{HF}$. With this adjustment, the BornOppenheimer dissociation energies of HOF are in excellent agreement with the experimental values recalculated from the new recommendation for the $\Delta H_{f, 0 \mathrm{~K}}^{\circ}$ of $\mathrm{HOF}$ given above. Considering the fact that $\operatorname{CCSD}(\mathrm{T})$ calculations employing large basis sets have been known to accurately reproduce bond dissociation energies in a large number of cases, ${ }^{49-51}$ the agreement between the calculated and experimental $D_{\mathrm{e}}$ values of $\mathrm{HOF}$ in Table 1 lend support to the recommended $0 \mathrm{~K}$ enthalpy of formation of HOF we have presented above.

We now comment on the two exceptions to the excellent agreement between calculated and experimental $D_{\mathrm{e}}$ values mentioned above. The first is due to the apparent difficulty of $\operatorname{CCSD}(\mathrm{T})$ to accurately recover the bond energy of the OF molecule, ${ }^{52}$ even when $\mathrm{CV}$ and SR corrections are included. As discussed below, the second is directly related to the error in the $\mathrm{OF}$ bond energy and is reflected in a relatively large discrepancy between the calculated and experimental $D_{\mathrm{e}}$ values for the process $\mathrm{HOF} \rightarrow \mathrm{H}\left({ }^{2} S\right)+\mathrm{OF}\left({ }^{2} \Pi\right)$.

The difficulty faced by $\operatorname{CCSD}(\mathrm{T})$ and MRCI $+\mathrm{Q}$ calculations in reproducing the bond dissociation energy of $\mathrm{ClO}^{53,54}$ and $\mathrm{BrO}^{55}$ are well-known. It appears that the OF molecule poses similar difficulties for these methods. Multiple reports have indicated that the $\mathrm{O}-\mathrm{F}$ bond is among the more challenging chemical bonds for other single determinant ab initio methods. ${ }^{9,56-58}$ The rather large discrepancy between the calculated and experimental $D_{\mathrm{e}}$ values for the process $\mathrm{HOF} \rightarrow$ $\mathrm{H}\left({ }^{2} S\right)+\mathrm{OF}\left({ }^{2} \Pi\right)$ is, therefore, easily explained in terms of the limited accuracy of the $D_{\mathrm{e}}$ value for OF from the calculations. For example, the difference between the $\operatorname{CCSD}(\mathrm{T}) / \mathrm{CBS}$ and experimental $D_{\mathrm{e}}$ values for OF $(1.29 \mathrm{kcal} / \mathrm{mol})$ is nearly exactly sufficient to account for the discrepancy between the CCSD(T)/CBS and experimental $D_{\mathrm{e}}(1.27 \mathrm{kcal} / \mathrm{mol})$ for $\mathrm{HOF} \rightarrow \mathrm{H}\left({ }^{2} S\right)$ $+\mathrm{OF}\left({ }^{2} \Pi\right)$. Despite these remarks, it should be noted that the experimental $D_{\mathrm{e}}$ value for OF has a significant error bar of $\pm 2.26 \mathrm{kcal} / \mathrm{mol}$. The ab initio results from CCSD(T)/CBS and higher levels of treatment are within this error limit.

We comment on one more item related to the dissociation energies of diatomic molecules from Table 1: the tendency for $\operatorname{CCSD}(\mathrm{T})$ calculations, when extrapolated to the CBS limit, to overestimate the $D_{\mathrm{e}}$ of HF. The tendency of large basis set $\operatorname{CCSD}(\mathrm{T})$ calculations to overestimate the $D_{\mathrm{e}}$ for this particular molecule is already known, for example, from the calculations of Feller, Peterson, and co-workers, ${ }^{49-51}$ where different functional forms were used for the extrapolation to the CBS limit. Therefore, our results are in keeping with those from several meticulous analyses of atomization energies of small molecules.

The enthalpies of reaction at $0 \mathrm{~K}, \Delta H_{r, 0 \mathrm{~K}}^{\circ}$, can be calculated from these $D_{\mathrm{e}}$ values simply by including the zero-point energies at each level of theory, i.e., by examining the spectroscopic dissociation energies, $D_{0}$. The harmonic frequencies and the anharmonic constants necessary for calculating these quantities are presented next. However, we mention here that the $\Delta H_{r, 0 \mathrm{~K}}^{\circ}$ $=D_{0}(\mathrm{HO}-\mathrm{F})$ for eq 6 calculated from the $D_{\mathrm{e}}$ values in Table 1 and the zero-point energies of $\mathrm{HOF}$ and $\mathrm{OH}$ turn out to be $47.54,47.64$, and $47.57 \mathrm{kcal} / \mathrm{mol}$, respectively, at the CBS, CBS $+\mathrm{CV}$, and CBS $+\mathrm{CV}+\mathrm{SR}$ levels of theory. These are in better agreement with the experimental value of $47.34 \pm 0.23$ $\mathrm{kcal} / \mathrm{mol}$ (see discussion following eq 6 , above) than the 48.4 $\mathrm{kcal} / \mathrm{mol}^{30}$ obtained from the G1 theory ${ }^{59}$ or a more recent estimate $^{60}$ of $48.11 \mathrm{kcal} / \mathrm{mol}$ from the $\mathrm{G} 2$ theory. ${ }^{61}$

III.B. Spectroscopic Constants. Table 2 summarizes the spectroscopic constants of the diatomic molecules at the various levels of theory used in this work. The spectroscopic constants for $\mathrm{HF}$ and $\mathrm{OH}$ are compared to the experimental values tabulated by Huber and Herzberg ${ }^{62,63}$ while those of OF are compared to the recent reevaluation of the spectroscopic properties of OF by Miller and Brouin ${ }^{28}$ from high-resolution spectroscopic data. It is seen that the CCSD(T) calculations (allelectrons correlated) overestimate the frequencies of all three diatomic species by just $5-15 \mathrm{~cm}^{-1}$.

The geometric parameters of HOF are presented in Table 3 where they are compared with the experimental values of Halonen and $\mathrm{Ha}^{20}$ It is seen that the higher-level calculations are able to reproduce the geometry of the molecule to within the $1 \sigma$ error estimates from the analysis by Halonen and Ha. This includes the HOF bond angle, which is consistently larger than the Halonen and Ha value by $\sim 0.2-0.5^{\circ}$ for all treatments beyond the CCSD(T)/AVDZ level. This is also consistent with the results obtained by Breidung et al. ${ }^{24}$ where the optimum 
TABLE 2. Spectroscopic Constants for the Diatomic Molecules ${ }^{a}$

\begin{tabular}{|c|c|c|c|c|c|c|c|}
\hline & AVDZ & AVTZ & AVQZ & CBS & $\mathrm{CBS}+\mathrm{CV}$ & $\mathrm{CBS}+\mathrm{CV}+\mathrm{SR}$ & $\operatorname{expt}^{b}$ \\
\hline \multicolumn{8}{|c|}{$\mathrm{HF}$} \\
\hline$r_{\mathrm{e}}$ & 0.9241 & 0.9210 & 0.9177 & 0.9156 & 0.9151 & 0.9151 & 0.9168 \\
\hline$\omega_{\mathrm{e}}$ & 4080.76 & 4124.69 & 4141.59 & 4152.46 & 4156.63 & 4153.68 & 4138.33 \\
\hline$\omega_{\mathrm{e}} x_{\mathrm{e}}$ & 97.01 & 85.57 & 87.72 & 89.74 & 89.83 & 89.81 & 89.881 \\
\hline$B_{\mathrm{e}}$ & 20.6259 & 20.7670 & 20.9152 & 21.0121 & 21.0356 & 21.0330 & 20.9558 \\
\hline$\alpha_{\mathrm{e}}$ & 0.8297 & 0.7617 & 0.7840 & 0.8017 & 0.8027 & 0.8030 & 0.7981 \\
\hline \multicolumn{8}{|c|}{ OF } \\
\hline$r_{\mathrm{e}}$ & 1.3783 & 1.3601 & 1.3536 & 1.3498 & 1.3482 & 1.3487 & 1.3542 \\
\hline$\omega_{\mathrm{e}}$ & 989.67 & 1054.42 & 1063.87 & 1068.41 & 1070.30 & 1068.55 & 1053.01 \\
\hline$\omega_{\mathrm{e}} x_{\mathrm{e}}$ & 10.13 & 10.38 & 10.21 & 10.14 & 10.14 & 10.13 & 9.919 \\
\hline$B_{\mathrm{e}}$ & 1.02177 & 1.04945 & 1.05948 & 1.06548 & 1.06802 & 1.06716 & 1.0587 \\
\hline$\alpha_{e}$ & 0.0136 & 0.0129 & 0.0132 & 0.0134 & 0.0134 & 0.0134 & 0.0133 \\
\hline \multicolumn{8}{|c|}{$\mathrm{OH}$} \\
\hline$r_{\mathrm{e}}$ & 0.9796 & 0.9733 & 0.9707 & 0.9691 & 0.9683 & 0.9684 & 0.9697 \\
\hline$\omega_{\mathrm{e}}$ & 3683.65 & 3717.94 & 3738.88 & 3752.19 & 3757.32 & 3754.84 & 3737.76 \\
\hline$\omega_{\mathrm{e}} x_{\mathrm{e}}$ & 87.12 & 80.87 & 83.09 & 84.81 & 84.89 & 84.88 & 84.881 \\
\hline$B_{\mathrm{e}}$ & 18.5299 & 18.7699 & 18.8724 & 18.9335 & 18.9618 & 18.9597 & 18.9102 \\
\hline$\alpha_{e}$ & 0.7288 & 0.7058 & 0.7164 & 0.7242 & 0.7251 & 0.7256 & 0.7242 \\
\hline
\end{tabular}

TABLE 3. Geometric Parameters of $\mathrm{HOF}^{a}$

\begin{tabular}{|c|c|c|c|c|c|c|c|}
\hline & AVDZ & AVTZ & AVQZ & CBS & $\mathrm{CBS}+\mathrm{CV}$ & $\mathrm{CBS}+\mathrm{CV}+\mathrm{SR}$ & $\operatorname{expt}^{20}$ \\
\hline $\mathrm{E}(\mathrm{au})$ & -175.203601 & -175.351315 & -175.397370 & -175.423402 & -175.531273 & -175.670112 & \\
\hline$r_{\mathrm{OH}}(\AA)$ & 0.9759 & 0.9703 & 0.9676 & 0.9660 & 0.9653 & 0.9654 & $0.9657(16)$ \\
\hline$r_{\mathrm{OF}}(\AA)$ & 1.4640 & 1.4418 & 1.4357 & 1.4322 & 1.4304 & 1.4311 & $1.4350(31)$ \\
\hline$\theta_{\mathrm{HOF}}(\mathrm{deg})$ & 97.18 & 97.74 & 97.89 & 97.98 & 98.04 & 98.00 & $97.54(50)$ \\
\hline
\end{tabular}

${ }^{a}$ Bond lengths are in $\AA$, and angles are in degrees. Experimental uncertainties shown in parentheses are $\pm 1 \sigma$ in the least-significant digits.

TABLE 4. Harmonic and Fundamental Vibrational Frequencies of HOF (in $\left.\mathrm{cm}^{-1}\right)^{a}$

\begin{tabular}{|c|c|c|c|c|c|c|c|}
\hline & AVDZ & AVTZ & AVQZ & CBS & $\mathrm{CBS}+\mathrm{CV}$ & $\mathrm{CBS}+\mathrm{CV}+\mathrm{SR}$ & expt \\
\hline$\omega_{\mathrm{s}}$ & 855.8 & 914.6 & 919.7 & 921.2 & 923.0 & 921.4 & $916.84 \pm 19^{b}$ \\
\hline$\omega_{\mathrm{b}}$ & 1366.0 & 1390.2 & 1399.0 & 1404.2 & 1406.5 & 1405.1 & $1396.22 \pm 7.0^{b}$ \\
\hline$\omega_{\mathrm{a}}$ & 3718.8 & 3745.5 & 3765.2 & 3778.03 & 3783.7 & 3780.9 & $3763.95 \pm 4.6^{b}$ \\
\hline$v_{\mathrm{s}}$ & 826.8 & 886.3 & 892.0 & 893.8 & 895.6 & 893.8 & $889.1^{c}$ \\
\hline$v_{\mathrm{b}}$ & 1321.2 & 1346.3 & 1355.1 & 1360.4 & 1362.6 & 1361.1 & $1353.4^{c}$ \\
\hline$\nu_{\mathrm{a}}$ & 3524.4 & 3564.6 & 3579.3 & 3588.3 & 3593.8 & 3591.0 & $3577.9^{c}$ \\
\hline
\end{tabular}

${ }^{a}$ Experimental uncertainties are $\pm 1 \sigma .{ }^{b}$ Reference 20. ${ }^{c}$ Reference 22.

TABLE 5. Rotational Constants of HOF in $\mathrm{cm}^{-1}$

\begin{tabular}{|c|c|c|c|c|c|c|c|}
\hline & AVDZ & AVTZ & AVQZ & CBS & $\mathrm{CBS}+\mathrm{CV}$ & $\mathrm{CBS}+\mathrm{CV}+\mathrm{SR}$ & $\operatorname{expt}^{21,22}$ \\
\hline$A_{\mathrm{e}}$ & 19.20810 & 19.50288 & 19.62923 & 19.70560 & 19.74460 & 19.73580 & 19.66380 \\
\hline$B_{\mathrm{e}}$ & 0.86560 & 0.89146 & 0.89889 & 0.90320 & 0.90530 & 0.90440 & 0.90026 \\
\hline$C_{\mathrm{e}}$ & 0.82830 & 0.85246 & 0.85950 & 0.86360 & 0.86560 & 0.86480 & 0.86084 \\
\hline
\end{tabular}

TABLE 6. Centrifugal Distortion Constants of $\mathrm{HOF}$ in $\mathrm{MHz}$

\begin{tabular}{lrrrrrrr}
\hline & AVDZ & AVTZ & AVQZ & CBS & CBS + CV & \multicolumn{2}{c}{ CBS + CV + SR } \\
\hline$D_{\mathrm{J}}$ & 0.0955 & 0.0911 & 0.0923 & 0.0933 & 0.0935 & 0.0936 & 0.09337 \\
$D_{\mathrm{JK}}$ & 2.2828 & 2.3254 & 2.3671 & 2.3945 & 2.4068 & 2.4034 & 2.387 \\
$D_{\mathrm{K}}$ & 70.8423 & 74.2277 & 75.3437 & 75.9935 & 76.4119 & 76.2804 & 75.125
\end{tabular}

geometry was obtained at the $\operatorname{CCSD}(\mathrm{T})$ level of theory using analytical derivatives.

The calculated harmonic and fundamental frequencies of HOF are tabulated in Table 4. The "experimental" harmonic frequencies shown for HOF were deduced from the experimental fundamental frequencies, together with calculated anharmonicity constants by Halonen and $\mathrm{Ha},{ }^{20}$ and have fairly large uncertainties. The CBS and higher levels of treatment have a tendency to slightly overestimate the fundamental frequencies. The CCSD(T)/AVQZ frequencies reported in Table 4 are very close to the $\operatorname{CCSD}(\mathrm{T}) / \mathrm{cc}-\mathrm{pVQZ}$ results of Breidung et al., ${ }^{24}$ who evaluated the frequencies from analytical second derivatives of the $\operatorname{CCSD}(T)$ wave function. The calculated fundamental frequencies are also in excellent agreement with the experimental results shown, the average difference for the three
$\mathrm{CBS}+\mathrm{CV}+\mathrm{SR}$ frequencies being of the order of $0.5 \%$ relative to the experimental values.

The rotational constants of HOF are presented in Table 5 and the centrifugal distortion constants in Table 6. The rather small differences between the calculated and experimental results can be attributed to the tendency for the calculated $\mathrm{OF}$ bond to be slightly too short $(\sim 0.004 \AA)$, as well as the vibrational effects on the centrifugal distortion constants, which we ignore.

The anharmonicity constants and rotation-vibration couplings are presented in Table 7 . The calculations are able to reproduce the two experimentally known anharmonicity constants of HOF within a fraction of a wavenumber. However, there are significant differences between some of the calculated and experimental rotation-vibration coupling constant, $\alpha$. The 
TABLE 7. Anharmonicity (in $\mathrm{cm}^{-1}$ ) and Vibration-Rotation Constants of HOF (in $\left.\mathbf{1 0}^{-3} \mathrm{~cm}^{-1}\right)^{a}$

\begin{tabular}{|c|c|c|c|c|c|c|c|}
\hline & AVDZ & AVTZ & AVQZ & CBS & $\mathrm{CBS}+\mathrm{CV}$ & $\mathrm{CBS}+\mathrm{CV}+\mathrm{SR}$ & $\operatorname{expt}^{b}$ \\
\hline$x(a, a)$ & -88.37 & -82.25 & -84.60 & -86.39 & -86.47 & -86.47 & \\
\hline$x(b, b)$ & -9.21 & -9.66 & -9.52 & -9.42 & -9.44 & -9.46 & $-9.942(2)^{c}$ \\
\hline$x(s, s)$ & -8.96 & -9.01 & -8.68 & -8.49 & -8.47 & -8.51 & \\
\hline$x(a, b)$ & -32.84 & -30.73 & -31.20 & -31.59 & -31.60 & -31.65 & \\
\hline$x(a, s)$ & -2.41 & -2.07 & -2.25 & -2.38 & -2.38 & -2.44 & \\
\hline$x(b, s)$ & -19.88 & -18.48 & -18.48 & -18.56 & -18.54 & -18.64 & $-18.502(2)^{c}$ \\
\hline$\alpha(a, A)$ & 739.20 & 716.49 & 729.70 & 739.16 & 740.53 & 741.01 & 738.733(9) \\
\hline$\alpha(b, A)$ & -451.94 & -467.31 & -471.45 & -473.81 & -476.19 & -475.40 & $-499.595(9)$ \\
\hline$\alpha(s, A)$ & 22.56 & 22.92 & 23.77 & 24.47 & 24.65 & 24.73 & 19.301(11) \\
\hline$\alpha(a, B)$ & 0.80 & 0.63 & 0.66 & 0.68 & 0.69 & 0.69 & $0.3834(8)$ \\
\hline$\alpha(b, B)$ & 4.74 & 3.86 & 3.86 & 3.89 & 3.88 & 3.91 & $3.7816(6)$ \\
\hline$\alpha(s, B)$ & 11.12 & 10.80 & 10.88 & 10.96 & 10.99 & 11.00 & $11.1685(22)$ \\
\hline$\alpha(a, C)$ & 1.89 & 1.71 & 1.76 & 1.80 & 1.81 & 1.82 & $1.6223(8)$ \\
\hline$\alpha(b, C)$ & 7.71 & 7.00 & 7.03 & 7.07 & 7.07 & 7.10 & $7.2037(6)$ \\
\hline$\alpha(s, C)$ & 10.84 & 10.60 & 10.69 & 10.78 & 10.80 & 10.81 & $10.9837(22)$ \\
\hline
\end{tabular}
21.

${ }^{a}$ Experimental uncertainties shown in parentheses are $\pm 1 \sigma$ in the least-significant digits. ${ }^{b}$ Reference 22 unless otherwise indicated. ${ }^{c}$ Reference

TABLE 8. The Quartic Force Field for HOF in Internal Coordinates in aJ $a_{0}{ }^{-\left(n_{1}+n_{2}\right)} \operatorname{rad}^{-n_{3}}(1 \mathrm{aJ}=1 \mathrm{mdyn} \AA)^{a}$

\begin{tabular}{|c|c|c|c|c|c|c|c|}
\hline$n_{1} n_{2} n_{3}$ & ref 24 & AVTZ & AVQZ & CBS & $\mathrm{CBS}+\mathrm{CV}$ & $\mathrm{CBS}+\mathrm{CV}+\mathrm{SR}$ & empirical $^{20}$ \\
\hline 200 & 8.000 & 7.827359 & 7.910119 & 7.964132 & 7.988109 & 7.976437 & $7.898(44)$ \\
\hline 020 & 4.723 & 4.590851 & 4.64969 & 4.670939 & 4.690732 & 4.672575 & $4.634(50)$ \\
\hline 002 & 0.998 & 0.990743 & 0.99745 & 1.001499 & 1.002958 & 1.001268 & $0.9906(54)$ \\
\hline 110 & -0.139 & -0.13468 & -0.13773 & -0.14046 & -0.14094 & -0.14075 & -0.135 \\
\hline 101 & -0.027 & -0.03293 & -0.03151 & -0.03059 & -0.02973 & -0.03036 & -0.037 \\
\hline 011 & 0.572 & 0.550296 & 0.561888 & 0.568789 & 0.571587 & 0.569158 & $0.572(38)$ \\
\hline 300 & -56.276 & -54.3741 & -55.4265 & -56.1584 & -56.3612 & -56.2978 & -54.366 \\
\hline 030 & -31.212 & -29.8537 & -30.2942 & -30.512 & -30.6675 & -30.5575 & $-30.87(30)$ \\
\hline 003 & -0.598 & -0.59544 & -0.60326 & -0.60792 & -0.61401 & -0.61025 & $-0.619(51)$ \\
\hline 210 & 0.801 & 0.747268 & 0.771159 & 0.7896 & 0.78951 & 0.784401 & 0.844 \\
\hline 120 & -0.417 & -0.36393 & -0.39149 & -0.40483 & -0.41234 & -0.41796 & -0.396 \\
\hline 201 & -0.060 & -0.0484 & -0.03794 & -0.03008 & -0.03144 & -0.03382 & -0.080 \\
\hline 021 & -2.628 & -2.58022 & -2.61402 & -2.63527 & -2.64557 & -2.64037 & $-2.736(48)$ \\
\hline 111 & -0.211 & -0.20894 & -0.21198 & -0.21289 & -0.2171 & -0.21319 & -0.245 \\
\hline 102 & -0.271 & -0.3028 & -0.30916 & -0.31394 & -0.31337 & -0.31416 & -0.236 \\
\hline 012 & -1.910 & -1.9019 & -1.91086 & -1.91805 & -1.91854 & -1.91952 & $-1.859(12)$ \\
\hline 400 & 348.09 & 339.7102 & 346.0883 & 350.3262 & 351.8754 & 351.8014 & 320.96 \\
\hline 040 & 148.18 & 140.0799 & 148.9171 & 154.6646 & 156.0977 & 155.2735 & $147(53)$ \\
\hline 004 & -0.42 & -0.27575 & -0.21586 & -0.16466 & -0.17237 & -0.16961 & $-0.473(1100)$ \\
\hline 310 & -0.01 & 0.00000 & -0.1668 & -0.27799 & -0.33359 & -0.47259 & -0.98 \\
\hline 130 & 6.77 & 8.22861 & 8.395407 & 8.528843 & 8.528844 & 8.278649 & 6.00 \\
\hline 220 & -3.22 & -3.11353 & -3.22473 & -3.30256 & -3.30256 & -3.34148 & -1.99 \\
\hline 301 & -0.62 & -1.05953 & -0.9248 & -0.83053 & -0.84727 & -0.9751 & 0.25 \\
\hline 031 & 5.91 & 6.744007 & 6.809863 & 6.866182 & 6.815165 & 6.946736 & 5.60 \\
\hline 211 & -0.12 & -0.13454 & -0.10089 & -0.07734 & -0.09414 & -0.11264 & -0.08 \\
\hline 121 & 1.62 & 1.799523 & 1.866407 & 1.919975 & 1.919812 & 1.941791 & 1.96 \\
\hline 202 & -0.61 & -0.70506 & -0.68429 & -0.67595 & -0.68094 & -0.66923 & -0.50 \\
\hline 022 & 3.06 & 3.117164 & 3.191791 & 3.25139 & 3.243819 & 3.215378 & 3.61 \\
\hline 112 & 0.55 & 0.68593 & 0.743201 & 0.787793 & 0.793249 & 0.784869 & 0.59 \\
\hline 103 & 0.84 & 1.647493 & 1.707556 & 1.747063 & 1.747366 & 1.736356 & 0.72 \\
\hline 013 & 1.87 & 2.407367 & 2.456053 & 2.50224 & 2.499096 & 2.490849 & 2.56 \\
\hline
\end{tabular}

$a$ The indices $n_{1}, n_{2}$, and $n_{3}$ indicate the order of the derivative with respect to the internal coordinates $q_{1}=r_{\mathrm{OH}}-r_{\mathrm{OH}}^{e}, q_{2}=r_{\mathrm{OF}}-r_{\mathrm{OF}}^{e}$, and $q_{3}$ $=\theta_{\mathrm{HOF}}-\theta_{\mathrm{HOF}}^{e}$.

largest discrepancies appear to be in the constants $\alpha(s, A)$ and $\alpha(a, B)$. In these cases, the absolute differences between the CBS $+\mathrm{CV}+\mathrm{SR}$ constants and experimental values are $28 \%$ and $80 \%$, respectively. The $\operatorname{CCSD}(\mathrm{T}) / \mathrm{cc}-\mathrm{pVTZ}$ values for these constants obtained by Breidung et al. ${ }^{24}$ also have large absolute differences (18.3\% and $42.6 \%$, respectively) from the experimental values. Given the ability of high-level computational chemistry to accurately predict a wide range of molecular properties, and given the many difficulties of accurately analyzing experimental molecular spectra, it may not be entirely unreasonable to suspect that the experimental constants may be in error in at least these two cases.

The quartic force fields at each level of theory examined are calculated from SURFIT in terms of displacement coordinates $q_{1}=r_{\mathrm{OH}}-r_{\mathrm{OH}}^{e}, q_{2}=r_{\mathrm{OF}}-r_{\mathrm{OF}}^{e}$, and $q_{3}=\theta_{\mathrm{HOF}}-\theta_{\mathrm{HOF}}^{e}$, where the superscript " $e$ " indicates the equilibrium value, and are presented in Table 8. Since the CCSD(T)/AVDZ results are of limited interest, we have omitted them in favor of the CCSD(T) results of Breidung et al., ${ }^{24}$ in which the quadratic force constants were obtained at the CCSD(T)/cc-pVQZ level and the cubic and quartic force constants at the CCSD(T)/cc-pVTZ level of theory using analytical second derivatives. The present results are comparable to those of Breidung et al. in most cases, but a few significant differences do exist, e.g., the force constants $(2,0,1),(0,0,4),(3,1,0),(1,3,0),(3,0,1),(0,3,1)$, and $(1,2,1)$. However, the present results shown in Table 8 indicate that most of these are very sensitive to the basis set used, as well as corevalence correlation and scalar relativistic effects. For these reasons we believe that the present CBS $+\mathrm{CV}+\mathrm{SR}$ results for $(2,0,1),(0,0,4),(3,1,0),(3,0,1)$, and $(1,2,1)$ are probably the 
more reliable. Our $(1,3,0)$ and $(0,3,1)$ force constants are larger than those of Briedung et al. and cannot be easily explained by the larger basis sets and other corrections used in this work, and hence, these differences are perhaps due to artifacts in the fits to $a b$ initio data.

It is also essential to comment on the differences between the present results and the empirical force field of Halonen and $\mathrm{Ha},{ }^{20}$ which is also shown in Table 8. This empirical force field is based on a constrained least-squares fit to available experimental vibration-rotation data combined with several force constants that were fixed at the ab initio values available then. ${ }^{23}$ These ab initio force constants are easily recognized in Table 8 by the absence of uncertainties in the last digits, which are shown in parentheses in the cases of the experimentally obtained values. Comparing these experimental force constants to those calculated in this work, we note that the calculated force constants differ from the experimental values by less than $5 \%$, with the notable exception of $(0,0,4)$, in which case the CBS + $\mathrm{CV}+\mathrm{SR}$ value differs from the experimental value by about $64 \%$. The case of $(0,0,4)$ was discussed above, as being one among the seven instances in which our results differ significantly from those of Breidung et al. ${ }^{24}$ The value reported from $\mathrm{SCF} / \mathrm{TZP} / / \mathrm{CCSD} / \mathrm{TZP}$ calculations ${ }^{23}$ is -0.78 , which is also in considerable disagreement with the experimental value. The extreme sensitivity of this force constant to the basis set and corrections used is evident from the fact that, between the CCSD(T)/AVTZ and the CBS + CV + SR levels of treatment, the calculated value changes by more than $62 \%$ relative to the latter value. Note that the $(0,0,4)$ force constant depends on a fourth-order derivative with respect to the HOF angle. The presence of diffuse functions in the basis set has been found to improve the accuracy with which ab initio calculations reproduce the angular dependence of potential energy functions in the case of $\mathrm{HOCl}^{53}$ and $\mathrm{HOBr}{ }^{55}$ Therefore, we believe that the CCSD(T)/AVQZ and higher results of the present work may be more reliable, although the $\operatorname{CCSD}(\mathrm{T}) / \mathrm{VTZ}$ value of Breidung et al. is in better agreement with the experimental value in this case. In this context, it is important to remember that the values obtained for the experimental force constants depend on the values provided for the remaining force constants in the analysis. Since constrained minimization affects all force constants that are not frozen, it is possible that using the CBS + CV + SR results in the development of a new force field could improve the agreement between the calculated and the experimental value in this particular case and further improve the agreement in the remaining cases.

\section{Summary}

We have presented calculations at the $\operatorname{CCSD}(\mathrm{T})$ level of theory for the $X^{1} A^{\prime}$ electronic state of the HOF molecule and the atom-diatom fragments that correlate with it. The ab initio calculations using the aug-cc-pVnZ $(n=\mathrm{D}, \mathrm{T}, \mathrm{Q})$ basis sets of Dunning and co-workers were followed by explicit pointwise extrapolation to the complete basis set (CBS) limit and the inclusion of core-valence $(\mathrm{CV})$ and scalar relativistic (SR) corrections. These calculations have been shown to be capable of accurately reproducing the Born-Oppenheimer dissociation energies of most of the species examined, with the possible exception of the OF molecule, in which case the highest level of treatment (CBS + CV + SR) is still smaller by $1.35 \mathrm{kcal} /$ mol than the experimental value but within the rather large error bar of $\pm 2.26 \mathrm{kcal} / \mathrm{mol}$ associated with the experimental result. The $1.42 \mathrm{kcal} / \mathrm{mol}$ discrepancy between the experimental and calculated (CBS $+\mathrm{CV}+\mathrm{SR}$ ) $D_{\mathrm{e}}$ value for $\mathrm{HOF} \rightarrow \mathrm{H}+\mathrm{OF}$ can be directly attributed to this error.
Note that the JANAF value of $-22.8 \mathrm{kcal} / \mathrm{mol}$ for the $\Delta H_{f, 0 \mathrm{~K}}^{\circ}$ of HOF would have led to serious discrepancies between the calculated and experimental $D_{\mathrm{e}}$ values in the last four rows of Table 1 . The approach taken in this work-large basis set $\operatorname{CCSD}(\mathrm{T})$ calculations supplemented by the various corrections - has been well-tested and has been shown to yield extremely accurate results in a large number of cases. ${ }^{49-51}$ Therefore, the ab initio results lend support to the recommendation of $-20.02 \pm 0.25 \mathrm{kcal} / \mathrm{mol}$ for the $\Delta H_{f, 0 \mathrm{~K}}^{\circ}$ of $\mathrm{HOF}$ made above.

This work also reports calculations of the spectroscopic constants for the HOF molecule and the diatomic fragments, as well as a quartic force field for the HOF molecule at each level of theory and basis set employed, from analytic fits to a moderate number of ab initio energies spanning near-equilibrium geometries. The larger basis set $\operatorname{CCSD}(\mathrm{T})$ calculations and further corrections to them are shown to accurately reproduce the spectroscopic properties of the diatomic molecules. The calculated spectroscopic constants for the HOF molecule, especially those at the CCSD(T)/AVQZ and higher levels of treatment, are also found to be in good agreement with experimental results, with the exception of two rotationvibration coupling constants. In these cases, there are large absolute differences between the experimental values and the present results as well as those of Breidung et al. ${ }^{24}$ In each case, the present results are in fair agreement with those of ref 24. This observation, coupled with the well-documented ability of large basis set $\operatorname{CCSD}(\mathrm{T})$ calculations to accurately reproduce a wide range of molecular properties, suggests that the experimental results may need to be reevaluated in at least these two cases.

To the best of our knowledge, the empirical force field of Halonen and $\mathrm{Ha}^{20}$ is the only one for $\mathrm{HOF}$ that combines available experimental vibration-rotation data with the only correlated $a b$ initio calculations of force constants available at the time. ${ }^{23}$ The ab initio force constants incorporated into this force field were obtained at the CCSD/TZP//CCSD/TZP (quadratic and cubic) and SCF/TZP//CCSD/TZP (quartic) levels of theory. ${ }^{23}$ There is no doubt that the $\operatorname{CCSD}(\mathrm{T})$ results of Breidung et al. ${ }^{24}$ and the present results are more reliable because the perturbative inclusion of triple excitations in the CCSD(T) method represents a significant improvement over CCSD. Use of some of these force constants in the place of those of Thiel et al. ${ }^{23}$ along with available experimental data may lead to a more accurate empirical force field for HOF and, perhaps, even better agreement between the calculated force constants presented in this work and those that are determined by constrained minimization.

\section{References and Notes}

(1) Buenker, R. J.; Peyerimhoff, S. D. J. Chem. Phys. 1966, 45, 3682.

(2) Kim, H.; Sabin, J. R. Chem. Phys. Lett. 1973, 20, 215.

(3) Botschwina, P. Chem. Phys. 1979, 40, 33.

(4) Murell, J. N.; Carter, S.; Mills, I. M.; Guest, M. F. Mol. Phys. 1979, 37, 1199 .

(5) Frenking, G.; Koch, W. Chem. Phys. Lett. 1984, 105, 659.

(6) Francisco, J. S.; Zhao, Y.; Lester, W. A., Jr.; Williams, I. H. J. Chem. Phys. 1992, 96, 2861.

(7) Burke, P.; Koppel, I. A.; Rummel, A.; Trummal, A. J. Phys. Chem. 1995, 99, 1432.

(8) Lee, T. J.; Rice, J. E.; Dateo, C. E. Mol. Phys. 1996, 89, 1359

(9) Ventura, O. N.; Kieninger, M. Chem. Phys. Lett. 1996, 245, 488.

(10) Dressler, S.; Thiel, W. Chem. Phys. Lett. 1997, 273, 71.

(11) Helgaker, T.; Gauss, J.; Jøgersen, P.; Olsen, J. J. Chem. Phys. 1997, 106,6430 .

(12) Berski, S.; Lundell, J.; Latajka, Z.; Leszczynski, J. J. Phys. Chem. A 1998, 102, 10768. 
(13) Berski, S.; Silvi, B.; Latajka, Z.; Leszczynski, J. J. Chem. Phys. 1999, 111, 2542.

(14) Kim, H.; Pearson, E. F.; Appleman, E. H. J. Chem. Phys. 1972, $56,1$.

(15) Pearson, E. F.; Kim, H. J. Chem. Phys. 1972, 57, 4230.

(16) Lovas, F. J. J. Phys. Chem. Ref. Data 1978, 7, 1445.

(17) Appleman, E. H.; Kim, H. J. Chem. Phys. 1972, 57, 3272.

(18) Rock, S. L.; Pearson, E. F.; Appleman, E. H.; Norris, C. L.; Flygare,

W. H. J. Chem. Phys. 1973, 59, 3940.

(19) Bürger, H.; Pawelke, G.; Rahner, A.; Appleman, E. H.; Mills, I. M. J. Mol. Spectrosc. 1988, 128, 278.

(20) Halonen, L.; Ha, T.-K. J. Chem. Phys. 1988, 89, 4885.

(21) Bürger, H.; Pawelke, G.; Sommer, S.; Rahner, A.; Appleman, E. H.; Mills, I. M. J. Mol. Spectrosc. 1989, 136, 197.

(22) Bürger, H.; Pawelke, G.; Rahner, A.; Appleman, E. H.; Halonen, L. J. Mol. Spectrosc. 1989, 138, 346.

(23) Thiel, W.; Scuseria, G.; Schaffer, H. F., III; Allen, W. D. J. Chem. Phys. 1988, 89, 4965.

(24) Breidung, J.; Thiel, W.; Gauss, J.; Stanton, J. F. J. Chem. Phys. 1999, 110, 3687.

(25) Raghavachari, K.; Trucks, G. W.; Pople, J. A.; Head-Gordon, M. Chem. Phys. Lett. 1989, 157, 479.

(26) Purvis, G. D., III; Bartlett, R. J. J. Chem. Phys. 1982, 76, 1910

(27) Gauss, J.; Stanton, J. F. Chem. Phys. Lett. 1997, 276, 70.

(28) Miller, C. E.; Drouin, B. J. J. Mol. Spectrosc. 2001, 205, 312.

(29) Ruscic, B.; Wagner, A. F.; Harding, L. B.; Asher, R. L.; Feller, D.; Dixon, D. A.; Peterson, K. A.; Song, Y.; Qian, X.; Ng, C.-Y.; Liu, J.; Chen, W.; Schwenke, D. W. J. Phys. Chem. A 2002, 106, 2727.

(30) Pople, J. A.; Curtiss, L. A. J. Chem. Phys. 1989, 90, 2833.

(31) (a) Kendall, R. A.; Dunning, T. H., Jr.; Harrison, R. J. J. Chem. Phys. 1992, 96, 6796. (b) Woon D. E.; Dunning, T. H., Jr. J. Chem. Phys. 1993, 98, 1358

(32) Peterson, K. A.; Woon, D. E.; Dunning, T. H., Jr. J. Chem. Phys. 1994, 100, 7410 .

(33) Woon, D. E.; Dunning, T. H., Jr. J. Chem. Phys. 1995, 103, 4572

(34) (a) Gdanitz, R. J.; Ahlrichs, R. Chem. Phys. Lett. 1988, 143, 413. (b) Werner, H.-J.; Knowles, P. J. Theor. Chim. Acta 1990, 78, 175.

(35) MOLPRO is a package of ab initio programs written by H.-J. Werner and P. J. Knowles, with contributions from Almlöf, J.; Amos, R. D.; Bernhardsson, A.; Berning, A.; Celani, P.; Cooper, D. L.; Deegan, M. J. O.; Dobbyn, A. J.; Eckert, F.; Elbert, S. T.; Hampel, C.; Hetzer, G.; Korona, T.; Lindh, R.; Lloyd, A. W.; McNicholas, S. J.; Manby, F. R.; Meyer, W.; Mura, M. E.; Nicklass, A.; Palmieri, P.; Peterson, K. A.; Pitzer, R. M.; Pulay, P.; Rauhut, G.; Schütz, M.; Stoll, H.; Stone, A. J.; Tarroni, R.; Taylor, P. R.; Thorsteinsson, T.

(36) (a) Rittby, M.; Bartlett, R. J. J. Phys. Chem. 1988, 92, 3033. (b) Scuseria, G. E. Chem. Phys. Lett. 1991, 176, 27. (c) Knowles, P. J.; Hampel, C.; Werner, H.-J. J. Chem. Phys. 1994, 99, 5219.

(37) Carter, S.; Handy, N. C. J. Chem. Phys. 1987, 87, 4294.
(38) SURFIT is described in "Spektroskopische Eigenschaften aus electronischen Wellenfunktionen," Senekowitsch, J., Ph.D. Thesis, Johann Wolfgang Goethe Universitaet, Frankfurt am Main, Germany, 1988.

(39) Hoy, A. R.; Mills, I. M.; Strey, G. Mol. Phys. 1972, 24, 1265.

(40) Papousek, D.; Aliev, M. R. Molecular Vibrational Spectra: Theory and Applications of High-Resolution Infrared, Microwave, and Raman Spectroscopy of Polyatomic Molecules; Elsevier: Amsterdam, 1982.

(41) Moore, C. E. Atomic Energy Levels; NSRD-NBS 35, Office of Standard Reference Data, National Bureau of Standards: Washington, DC, 1971.

(42) Chase, M. W., Jr.; Davies, C. A.; Downey, J. R., Jr.; Frurip, D. J.; McDonald, R. A.; Syverud, A. N. J. Phys. Chem. Ref. Data 1985, 14, (Suppl. $1)$.

(43) Berkowitz, J.; Appleman, E. H.; Chupka, W. A. J. Chem. Phys. 1973, 58,1950 .

(44) Reference 43 does not specify the experimental error in this measurement, but we have assumed that it is equal to that in $\mathrm{HOF} \rightarrow \mathrm{HOF}^{+}$ $+\mathrm{e}^{-}, 12.71 \pm 0.01 \mathrm{eV}$, measured in the same experiment.

(45) Katsumata, S.; Lloyd, D. R. Chem. Phys. Lett. 1977, 45, 519.

(46) Weidmann, R. T.; Tonkyn, R. G.; White, M. G.; Wang, K.; McKoy, V. J. Chem. Phys. 1992, 97, 768,

(47) Ruscic, B.; Berkowitz, J. J. Chem. Phys. 1994, 101, 7995.

(48) It is possible that this calculation has a small error, because using the same numbers, we get $-19.7 \mathrm{kcal} / \mathrm{mol}$

(49) Feller, D.; Peterson, K. A. J. Chem. Phys. 1999, 110, 8384.

(50) Feller, D. J. Chem. Phys. 1999, 111, 4373.

(51) Feller, D.; Peterson, K. A.; de Jong, W. A.; Dixon, D. A. J. Chem. Phys. 2003, 118, 3510

(52) Chase, M. W. J. Phys. Chem. Ref. Data 1996, 25, 551. A $D_{0}$ value of $18030 \pm 800 \mathrm{~cm}^{-1}$ is recommended. This, combined with the spectroscopic constants and the $\mathrm{OF}\left({ }^{2} \Pi\right)$ spin-orbit coupling constant of $196.6284 \mathrm{~cm}^{-1}$ obtained in ref 28 , yields the experimental $D_{\mathrm{e}}$ value shown

(53) (a) Skokov, S.; Peterson, K. A.; Bowman, J. M. J. Chem. Phys. 1998, 109, 2662. (b) Peterson, K. A.; Skokov, S.; Bowman, J. M. J. Chem. Phys. 1999, 111, 7446

(54) Ramachandran, B.; Peterson, K. A. J. Chem. Phys., in press.

(55) Peterson, K. A. J. Chem. Phys. 2000, 113, 4598.

(56) Jensen, F. Chem. Phys. Lett. 1990, 169, 519.

(57) Tozer, D. J.; Handy, N. C.; Amos, R. D.; Pople, J. A.; Nobes, R. H.; Xie, Y.; Schaefer, H. F. Mol. Phys. 1993, 79, 777.

(58) Li, X. Z.; Paldus, J. J. Chem. Phys. 1996, 104, 9555

(59) Pople, J. A.; Head-Gordon, M.; Fox, D. J.; Raghavachari, K.; Curtiss, L. A. J. Chem. Phys. 1989, 90, 5622.

(60) Glukhovtsev, M. N.; Pross, A.; Radom, L. J. Phys. Chem. 1996 100,3498

(61) Curtiss, L. A.; Raghavachari, K.; Trucks, G. W.; Pople, J. A. J. Chem. Phys. 1991, 94, 7221.

(62) Huber, K. P.; Herzberg, G. Constants of Diatomic Molecules; Van Nostrand Reinhold: New York, 1979.

(63) Also available at http://webbook.nist.gov/chemistry. 